\title{
Artistic Tiling Using TilerPro
}

\author{
Nasir Al-Darwish* \\ Information and Computer Science Department, King Fahd University of Petroleum and Minerals, Dhahran, \\ Saudi Arabia. \\ * Corresponding author; Email: darwish@kfupm.edu.sa \\ Manuscript submitted July 3, 2016; accepted October 5, 2016. \\ doi: 10.17706/jsw.11.11.1102-1110
}

\begin{abstract}
TilerPro is a web-based software system for building symmetric tiling designs. With TilerPro, the user is able to quickly build and save a large number of aesthetic tiling designs. Graphics generation by TilerPro takes place entirely on the client-side using SVG with program code written in JavaScript. This paper gives an overview of the system.
\end{abstract}

Key words: Digital art, symmetry, tiling software, SVG graphics.

\section{Introduction}

This paper describes some features and uses of TilerPro software. TilerPro is a web-based system for generating sophisticated tiling designs that exhibit pleasant symmetry. Besides tiling designs, the type of graphic images produced by TilerPro can be useful for paintings, wall decorations, carpet, and textile fabrics. The images can also serve as eye-catching background graphics for posters and billboards.

TilerPro uses vector graphics and can save a tiling design to SVG (Scalable Vector Graphics) format - SVG (http://www.w3.org/Graphics/SVG/) is an XML-based graphics format supported by modern graphics tools and browsers. SVG has matured into an effective way for rendering 2-D graphics and is widely used by several web-based data visualization and charting tools including D3.js (http://d3js.org/). Unlike bitmap graphics, vector graphics objects have the advantage that they can be scaled to any size without being distorted. Furthermore, TilerPro encodes a tiling design as a short string of characters (from which the image can be reconstructed). Thus, the system can save thousands of tiling designs using a tiny amount of disk space. In the current version of TilerPro, the image data can be saved to "My Goodies" list (this uses the browser's local storage). Graphics processing by TilerPro takes place entirely on the client-side (i.e., the browser) mostly using SVG operations without using any third-party JavaScript library (except for Pan/Zoom function). TilerPro is best run with Mozilla FireFox or Google Chrome browsers because of some shortcomings in SVG implementation by Microsoft IE browser.

TilerPro relies on symmetric curves defined by some recursive mathematical functions based on a novel idea of extended Sierpinski's curves [1]. Symmetry is often associated with regularity (repetitiveness) that is somehow aesthetically pleasing. With geometric objects such as 2-D drawings or 3-D physical structures, symmetry is defined through geometric transformations such as scaling, reflection, and rotation. This is the case with extended Sierpinski's curves, as they exhibit reflection and rotational symmetry.

A tessellation (tiling) of a 2-D plane is a covering of the plane by one or more closed geometric shapes without any gaps or overlaps [2]. A tiling of the plane by copies of a single shape is known as isohedral tiling. A tiling that uses a regular polygon (an $n$-gon with equal sides, e.g. a triangle, a square or a hexagon) is 
known as a regular tiling. It is a proven fact that there are only three kinds of polygons which can fill a plane without gaps: triangles, squares and hexagons. Tessellations and artistic geometric patterns are prevalent in Islamic ornaments used to decorate medieval mosques and palaces. Because of their awesome beauty and sophistication, Islamic geometric patterns have been the focus of study by mathematicians in recent decades [3], [4]. In modern times, the work on tessellations by M. C. Escher has led to some interesting theoretical results [5], [6]. Mathematical foundation for symmetry and tiling can be found in the writings by Grünbaum and Shephard [3], Chavey [7], Abas and Salman [8], Horne [9], and Kaplan [10]. The writings by Darvas [11], Lu and Steinhardt [12], Krawczyk [13], Deger, K. O. and Deger, A. H. [14], and Ouyang, P., Zhao, W., and Huang, X. [15] shed light on some aspects of the ongoing research.

Modern graphics and drawing tools such as Inkscape (https://www.inkscape.org/) have built-in commands for filling and tiling a canvas with a selected shape but little in the way of easily creating a symmetric shape in the first place. Creating a symmetric design by hand is undoubtedly a tedious process, even for artists. This has driven some researchers to propose and develop graphics drawing systems with built-in aids for constructing symmetric drawings [16]-[22] (listed in chronological order). However, unlike TilerPro, none of the proposed systems is currently available for public use.

\section{Tiling with Symmetric Curves}

The tiling designs produced by TilerPro are based on regular tiling. The design starts with a basic square (or rectangular) shape that represents a single tile which is then laid out horizontally and vertically. Diamond (and Diamond-Alternating) tiling designs are also constructed by laying out square tiles.

Consider the curve shown in Fig. 1-left. Note that for the purpose of tiling, the curve's closed regions are filled (using even-odd rule) with a particular color. The curve (image) exhibits a rotational symmetry of order 4 (i.e., the shape coincides with itself with every 90-degree rotation around its center). It also exhibits reflection symmetry; the bottom half is a reflection of the top half, and, at the same time, the left half is a reflection of the right half. Furthermore, the shape is symmetric with respect to both diagonals. These characteristics hold for any square around the center of this image (as in the dotted square shown). As can be seen from Fig. 1-right, a regular tiling using this kind of curves will still exhibit the curve symmetries. In addition, the tiled area (assumed to be infinite in both the $\mathrm{x}$ and $\mathrm{y}$ directions) exhibits a translation symmetry (x-movement and/or $y$-movement by the side length). More compelling tiling designs, such as that shown in Fig. 2, are built by overlapping (coinciding the centers of the curves) two symmetric curves.
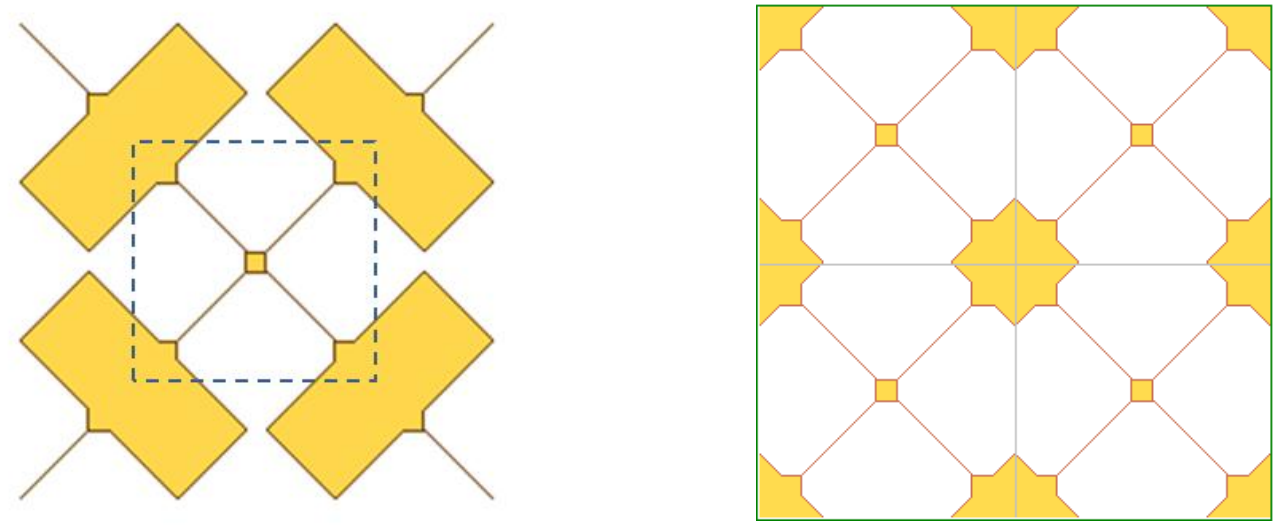

Fig. 1. A symmetric filled curve showing a square tile (area with dotted border) and its regular tiling (right figure). The tiling shows the 8-point Islamic star pattern.

The tiling designs produced by TilerPro are quite aesthetic. This can be attributed to several factors. First, 
each tile exhibits curves with rotational and reflection symmetries. Second, the filled regions of adjacent tiles mesh together exhibiting a new pattern that is repeated throughout the tiling. Third, TilerPro uses contrasting line and fill colors adding a color-symmetry dimension to the tiling.

TilerPro uses several independent parameters (options) that result in a large variety (hundreds of millions) of tiling designs. For example, when superimposing two curves, the tile is taken to be a central square (or a rectangle) of the image whose size can be varied by the user (this is specified using MarginX/Margin Y parameters). Another option allows the user to rotate one or both curves by a 45 -degree angle. One key parameter is Tile Type (Square, Diamond, Diamond-Alternating). Fig. 2, 3 and 4 show the different types of tiling designs as set by tile type. From a viewer standpoint, a diamond tiling (Fig. 3) shows one kind of a diamond tile throughout the design. On the other hand, a diamond-alternating (Fig. 4) tiling shows two kinds of diamond tiles laid out in alternate rows. Diamond tiling designs are actually built by laying out ordinary square tiles; this may be surprising but it will become apparent to the viewer when the "Grid" option is enabled.

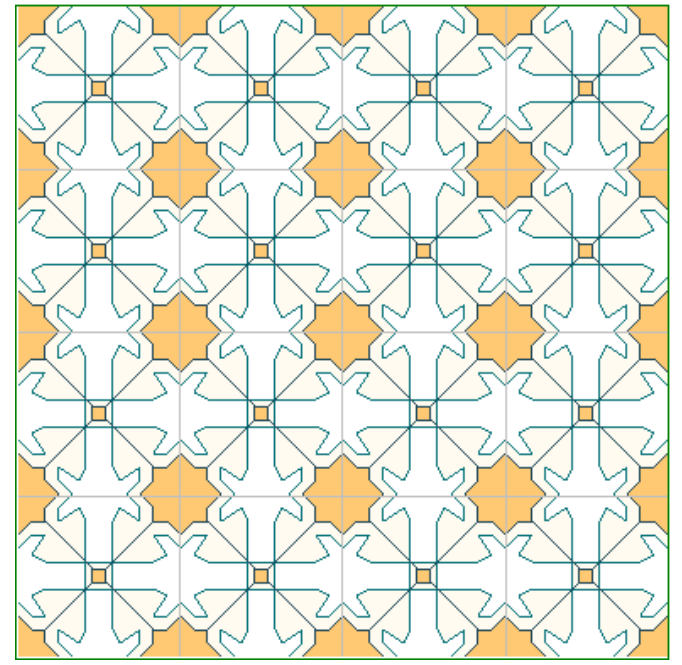

Fig. 2. Sample square tiling. name in my goodies list: Best104.

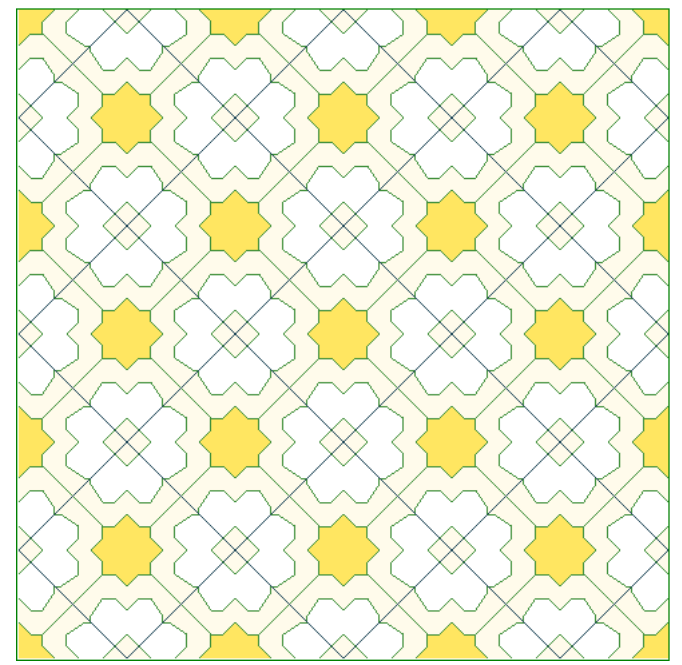

Fig. 3. Sample diamond tiling. To reproduce, paste the following string in the image name textbox and click load/edit image button: qFFFFFFFFvCDCACxCBBsCAsBxBCCCACxDq2q20q0q0q41913CqfffffbebwvFFBBBBsBAAABAsBxBBxBFxFq 2q20q0q0q41913Cqffffe661w2q80q4q2q2q0q0q2q2q2. 


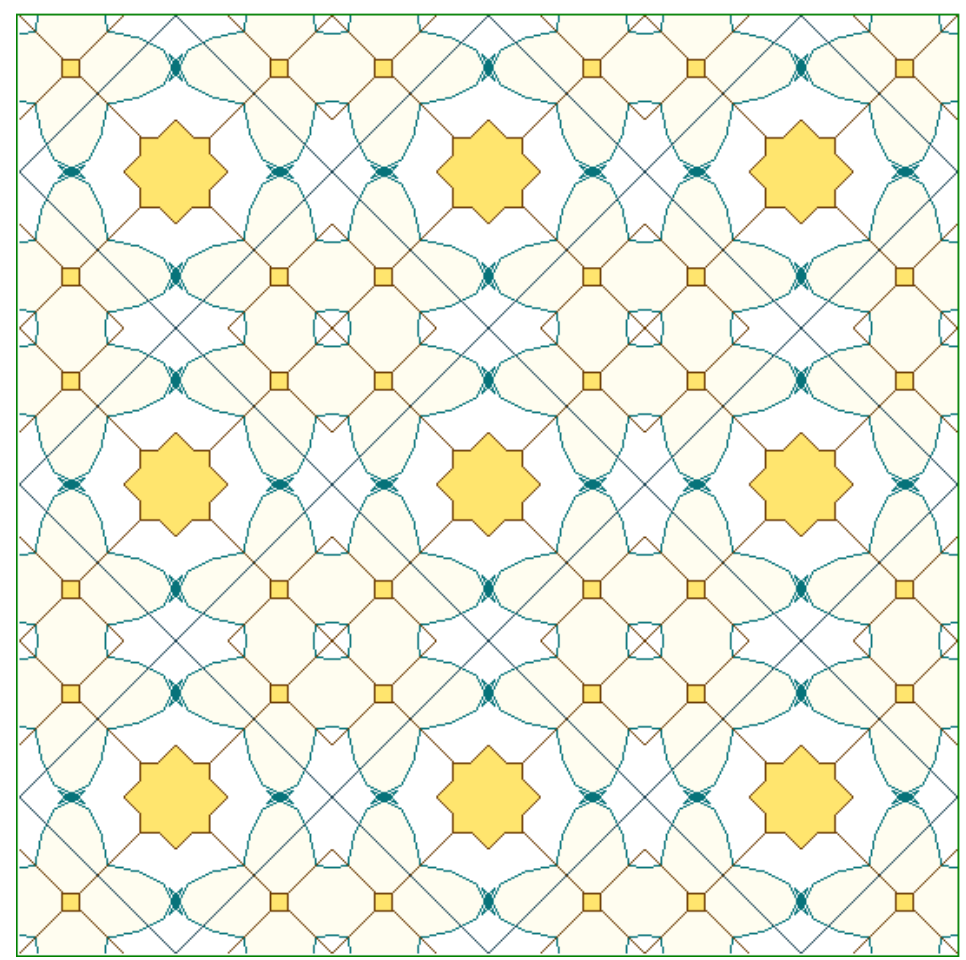

Fig. 4. Sample diamond-alternating tiling. name in my goodies list: Dimr1_1wowwow.

\section{TilerPro Web Interface}

TilerPro, like the majority of web applications, uses a form-based interface. Fig. 5 shows the main window for TilerPro. In the window, every time the user clicks Generate button, a new image (because First Curve is set to Random) will be built and displayed. The new image will comply with all the set options. For example, the user can change Tile Type to Diamond and then click Generate button to see that tiling designs that are generated will change from square tiling to diamond tiling.



Fig. 5. The main window of TilerPro.

The top part of the main window, which consists of the Tile Type row and Color Scheme row, is used to specify the properties of the generated images. The top row specifies the permanent properties of the 
generated images.

- The Tile Type option specifies the shape of the tile (Square, Diamond, Diamond-Alternating).

- The First Curve and Second Curve options are for selecting the first curve and optionally a second curve.

- The Level option is a parameter used by the algorithm that draws these curves and will affect the shape of the generated curve.

- The Rotate option allows for a 45-degree rotation of one or both of the curves.

- The Fill option controls the filling of closed areas that result from either curve.

- Finally, the Color Scheme option allows for selecting from some predefined schemes.

Table 1. Summarizes the Various Options That Can Be Set Through the Main Window

\begin{tabular}{|l|c|l|}
\hline \multicolumn{1}{|c|}{ Option } & Default Value & \multicolumn{1}{|c|}{ Other Possible Values } \\
\hline Tile Type & Square & Square, Diamond, Diamond Alt. \\
\hline First Curve & Random & Some other predefined curves \\
\hline Second Curve & Islamic & Some other predefined curves \\
\hline Level & Two & One, Three \\
\hline Fill & Both & None, First, Second, Both \\
\hline Rotate & None & None, First, Second, All \\
\hline Color Scheme & Vary Light1 & Fixed, Vary Light2, Vary Heavy \\
\hline Grid & Checked & Unchecked \\
\hline Frame & None & Half Tile, Full Tile \\
\hline Unit & 80 & $\begin{array}{l}\text { Used to control the curve drawing and as such it affects tile size; use a positive } \\
\text { number that is a multiple of } 16 .\end{array}$ \\
\hline MarginX/MarginY & 0 & $\begin{array}{l}\text { This option allows for a slight change to the curves and it may change the size of the } \\
\text { tile. Margin values can be in the range } 0-8 \text { (negative or positive). }\end{array}$ \\
\hline
\end{tabular}

Table 1 Options set through the main window of TilerPro.

\section{Command Buttons}

Generate Button: Use this button to build and generate images. Every time you click on this button, a new image is displayed in accordance with the chosen image properties (options). The honored options also include these options: Unit, Grid, MarginX, MarginY.

Load/Edit Image Button: Use this button to chnage some properties of the current image. The properties that can be changed are those shown in Load/Edit Image box. In addition, the user can enable (or disable) the Grid and Frame properties. Every time some of the image properties are changed, this button must be clicked for the desired effect to take place. Note: This button can be used to load an image using the image's internal name entered in Image Name textbox (as in the example in Fig. 3). The following is a description of the options used with the Load/Edit Image Button.

- Vary Line Color: If checked, then the line colors are randomly chosen from the predefined list of line colors (set by using the LineColors button).

- Vary Fill Color: If checked, then the fill colors are varied according to the color scheme chosen from the Color Scheme list. 
- Vary FO: This option allows the user to vary the order of fill when the Fill option is chosen as Fill-both.

- Unit: This is a measure in pixels related to the drawing unit of the curve. This option can be used to enlarge or shrink the tile. To avoid distorted shapes, Unit must be set to a multiple of 16. Note: The Unit value is saved as part of the image data (i.e., the image internal name). The tile width/height can also be changed in the Edit Layout window.

- MarginX/MarginY: This parameter affects the size of the tile but in a way different from the drawing unit. Because the tile is confined to a square area placed within the drawing canvas, this option allows for controlling the size of such a square. A positive $x$-margin [y-margin] will increase the width [height] of the tile. Likewise, a negative $x$-margin [y-margin] will decrease the width [height] of the tile. Note: In addition to affecting the size of the tile, the use of margin values will also affect the shape of the tile. To get rectangular (instead of square) tiles use margin values where MarginX $\neq$ MarginY.

Save Image Button: Use this button to add the current image to My Goodies list. You need to type in some description in the Image Desc box and then click on Save Image. The image will show at top of the goodies list. The list's information is saved to the browser's local storage and will be available when TilerPro is closed and then restarted. Note that the goodies list is sorted alphabetically when the application is started.

Load Image Button: Use this button to load an image from the My Goodies list, and display it as the current image. The chosen image will also be moved to the top of the list and thus it can be used as one of the source curves for building images (This is done by selection Top Goody value from First Curve or Second Curve lists).

Edit Layout Button: Use this button Use this button to show the Edit-Layout window (Fig. 6). The window allows the user to prepare a final image by setting the desired width/height of the tile (this will scale the tile) and the number of horizontal/vertical tiles and save the image as a disk file using a user chosen graphics format.

Line Colors Button: Using this button will show a popup window for specifying a set of colors that are used as curve-line colors for the generated tilings.

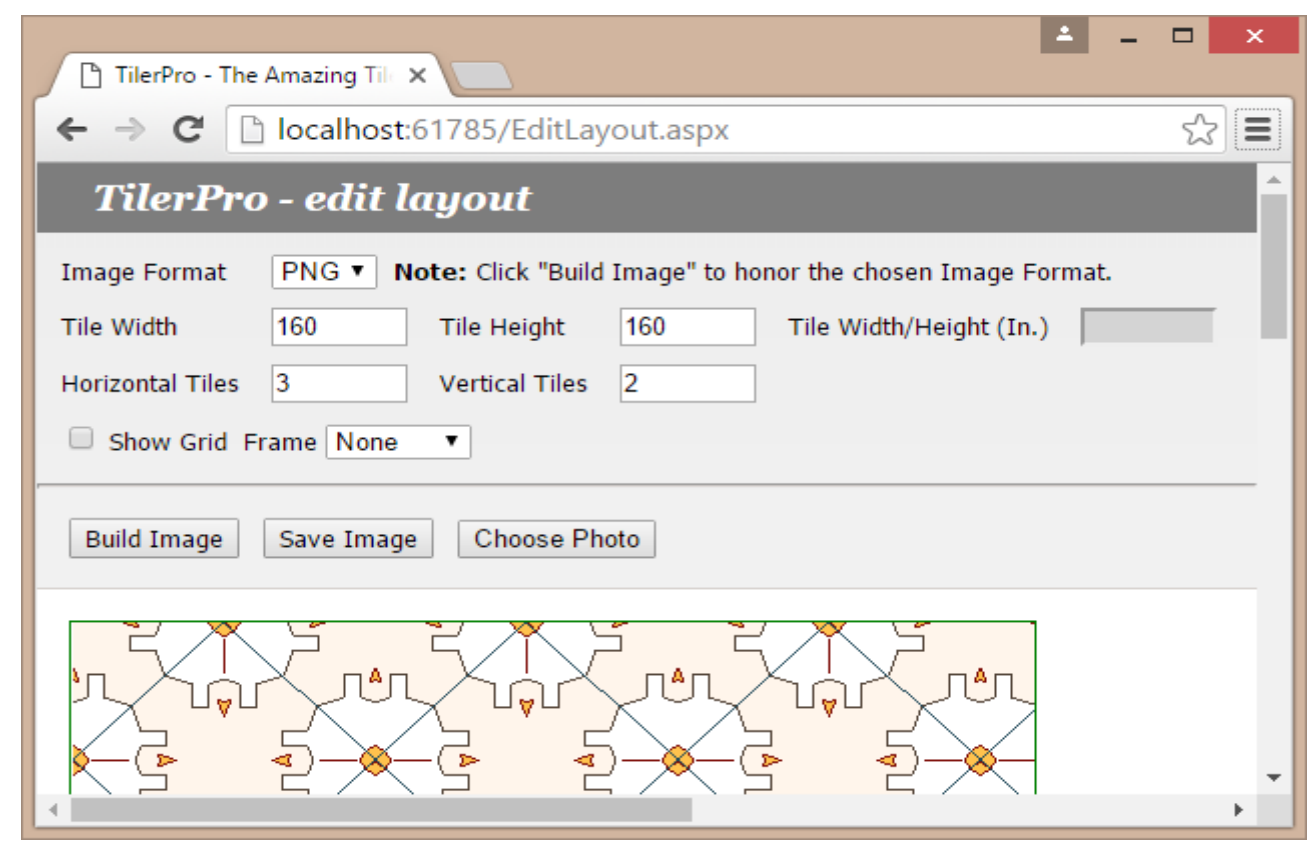

Fig. 6. The edit-layout window. 


\section{Sample Aesthetic Tilings}

Some aesthetic tilings can be obtained using the following option settings: Tile Type: Square (or Diamond), Level: two, First Curve: Random, Second Curve: any curve other than random. In particular, the combination of Random and Islamic curves produces nice tilings with the 8-point Islamic star normally found in Islamic ornaments (as the sample in Fig. 7) - see Al-Darwish [23] for more information. A number of such tilings (named as Islamic $<1 X X>$ ) have been saved to My Goodies list. Some of these tilings can be browsed at http://faculty.kfupm.edu.sa/ICS/darwish/1001Tiles/.

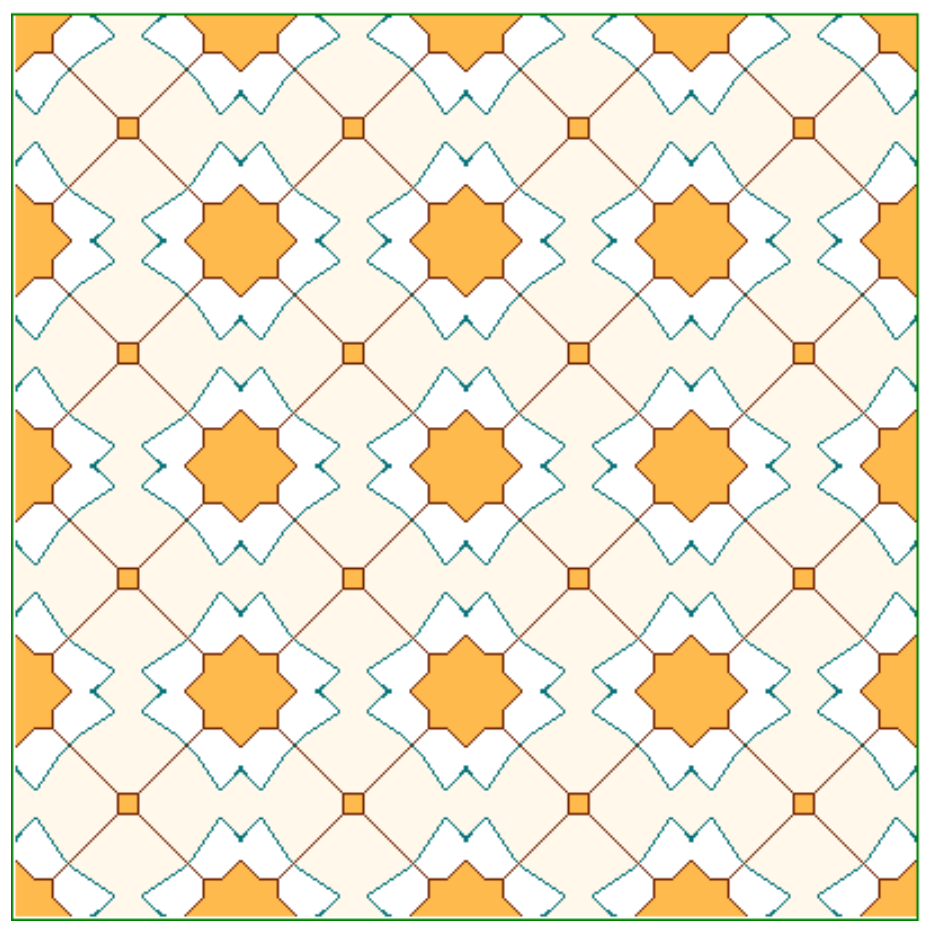

Fig. 7. Islamic square tiling. Name in My Goodies list: Best101.

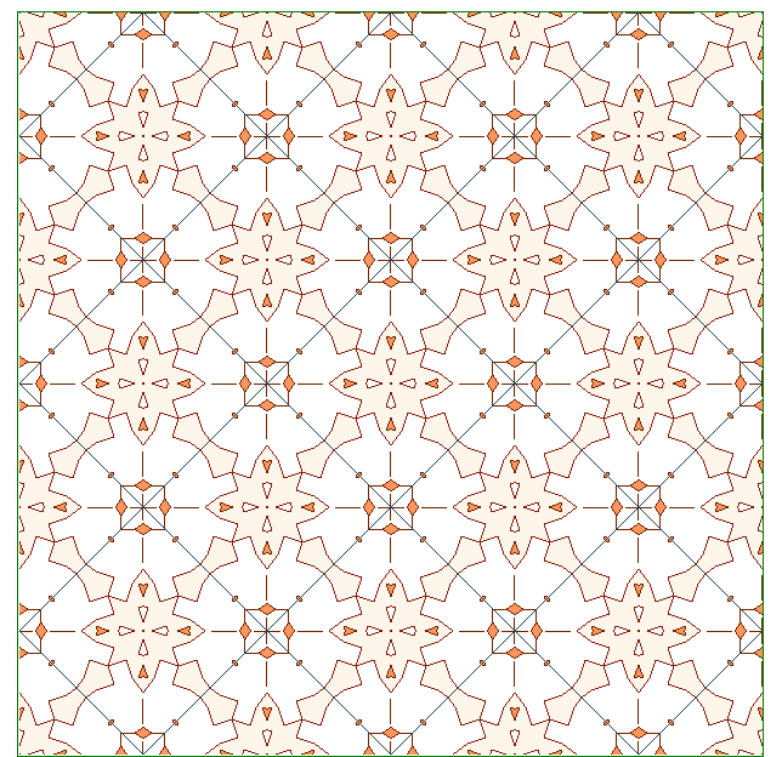

Fig. 8. StarArrow diamond tiling. Name in my goodies list: dimstararrowu96r2m-8_6.

Another set of aesthetic tilings (similar to that shown in Fig. 8 has been saved to My Goodies list with names beginning with "DimStarArrowU96R2M-8" (corresponding to option settings: TileType: Diamond, FirstCurve: Random, Second Curve: StarArrow, Unit: 96, Rotate: Second, MarginX/Y:-8). 
Finally, we should note that, as shown in Fig. 9, TilerPro (using Choose Photo button and Frame option in Edit-Layout window) can aid in creating artistic frames around pictures chosen by the user.

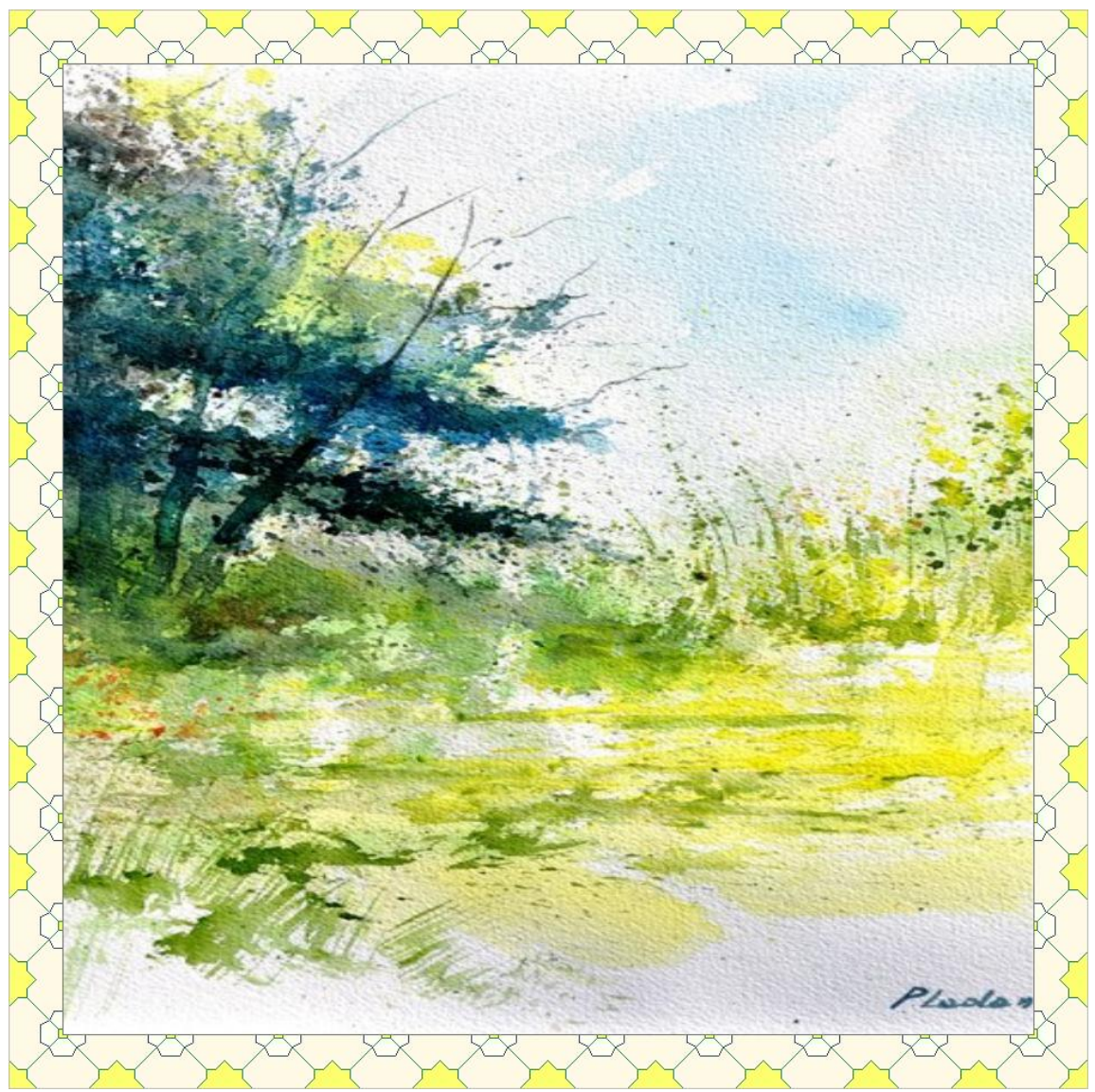

Fig. 9. A sample image with an artistic frame produced by TilerPro.

\section{Conclusion}

In this paper, we have given an overview of TilerPro software for generating tiling designs. It is hoped that interested users and researchers find it entertaining and inspiring. As a web application, TilerPro serves as another example of the utility of the SVG standard for browser-based 2-D graphics.

\section{Acknowledgment}

This work has a companion website currently available at http://tiler.gear.host/TilerStart.aspx. The author acknowledges the free hosting support provided by GearHost.

\section{References}

[1] Al-Darwish, N. (2012). Extended Sierpinski'S curve and tiling applications. International Journal of Web Applications, 4 (4).

[2] El-Said, I., Parman, A. (1976). Geometric Concepts in Islamic Art. World of Islam Festival Publishing Co.

[3] Grünbaum, B., \& Shephard, G. C. (1987). Tilings and Patterns. W.H. Freeman and Co., New York.

[4] Abas, S. J., \& Salman, A. S. (1995). Symmetries of Islamic Geometrical Patterns. World Scientific Publishing Co., New Jersey.

[5] Schattschneider, D. (2004). M. C. Escher : Visions of Symmetry. Thames \& Hudson, London.

[6] Abas, S. J. (2002). Islamic patterns: The spark in escher's genius. M.C.Escher's Legacy: A Centennial Celebration (pp. 100-112). New York, Springer Verlag. 
[7] Chavey, D. (1989). Tilings by regular polygons-II: A catalog of tilings. Computers \& Mathematics with Applications, 17(1), 147-165.

[8] Abas, S., \& Salman, A. (1992). Geometric and group-theoretic methods for computer graphics studies of Islamic symmetric patterns. Computer Graphics, 11(1), 43-53.

[9] Horne, C. E. (2000). Geometric Symmetry in Patterns and Tilings. CRC Press.

[10] Kaplan, C. S. (2009). Introductory Tiling Theory for Computer Graphics. Morgan \& Claypool.

[11] Darvas, G. (2007). Symmetry in geometrical decorative art. Cultural-historical and Ontological Aspects of Science-Arts Relations.

[12] Lu, P. J., \& Steinhardt, P. J. (2007). Decagonal and quasi-crystalline tilings in medieval Islamic architecture. Science, $\quad$ Retrieved $\quad$ August 2016, from http://physics.princeton.edu/ steinh/LuSteinhardt2007.pdf

[13] Krawczyk, R. J. (2011). Truchet tilings revisited. Proceedings of ISAMA 2011 (pp. 69-77).

[14] Deger, K. O., \& Deger, A. H. (2012). An application of mathematical tessellation method in interior designing. Procedia-Social and Behavioral Sciences, Retrieved August 5, 2016, from http://www.sciencedirect.com/science/article/pii/S1877042812032922

[15] Ouyang, P., Zhao, W., \& Huang, X. (2015). Beautiful math, Part 5: Colorful archimedean tilings from dynamical systems. IEEE Computer Graphics and Applications, 35(6), 90-96.

[16] Wong, M. T., Zongker, D. E., \& Salesin, D. H. (1998). Computer-generated floral ornament. Computer Graphics (SIGGRAPH 98), 423-434.

[17] Akleman, E., Chen, J., \& Meric, B. (2000). Intuitive and effective design of periodic symmetric tiles. Proceedings of ACM Multimedia 2000 (pp. 100-108).

[18] Kaplan, C. S. (2005). Islamic star patterns from polygons in contact. Graphics Interface 2005, ACM Proceedings of the International Conference Proceeding Series 112 (pp. 177-186).

[19] Liu, S. (2009). The generation system of textile pattern based on quasi-regular pattern theory. Information Engineering and Electronic Business.

[20] Qi, W. J., \& Li, X. Q. (2009). Example-based floral pattern generation. Proceedings of the Fifth International Conference on Image and Graphics (pp. 553-558).

[21] Izadi, A., Rezaei, M., \& Bastanfard, A. (2010). A computerized method to generate complex symmetric and geometric tiling patterns. Intelligent Computer Graphics 2010 (pp. 185-210).

[22] Alani, M., \& Barrios, C. (2015). A parametric metamorphosis of Islamic geometric patterns. Proceedings of the 20th International Conference of the Association for Computer-Aided Architectural Design Research in Asia (CAADRIA 2015) (pp. 593-602).

[23] Al-Darwish, N. (2012). Creative tiling: A story of 1000-and-1 curves. Acta Didactica Napocensia, 5(1).

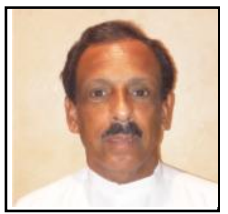

Nasir Al-Darwish is an associate professor with the Information and Computer Science Department, King Fahd University of Petroleum and Minerals (KFUPM), Saudi Arabia.

Recently, Dr. Al-Darwish developed TilerPro software - A web-based system for building symmetric tiling designs. 